\title{
THE POINT SPREAD FUNCTION VARIATIONS INSIDE WIDE-FIELD ASTONOMICAL IMAGES
}

\author{
Elena Anisimova*, Jan Bednář, Petr Páta \\ Czech Technical University in Prague, Faculty of Electrical Engineering, Dept. of Radioelectronics, Technická 2, \\ 166 27, Prague 6, Czech Republic \\ * corresponding author: anisiele@fel.cvut.cz
}

\begin{abstract}
The Point Spread Function (PSF) of the astronomical imaging system is usually approximated by a Gaussian or Moffat function. For simplification, the astronomical imaging system is considered to be time and space invariant. This means that invariable PSF within an exposed image is assumed. If real wide-field imaging systems are considered, this presumption is not fulfilled. In real systems, stronger optical aberrations are expected (especially coma) at greater distances from the center of the captured image. This impacts the efficiency of stellar astrometry and photometry algorithms, so it is necessary to know the PSF variation. In this paper, we perform the first step toward assigning PSF changes: we study the dependence of the Moffat function fitting parameters (FWHM and the atmospheric scattering coefficient $\beta$ ) on the position of a stellar object.
\end{abstract}

KEYWORDS: astronomy, wide-field imaging, wavelet transform, à trous, fitting.

\section{INTRODUCTION}

Lenses with a wide field of view (WFC - Wide-Field Camera, UWFC - Ultra Wide-Field Camera) are used in a wide range of applications in sky monitoring. Well-known applications of this kind of image analysis are for detecting new objects, e.g. novae, supernovae and AGN (Active Galactic Nuclei).

UWFC image data analysis is in general a very difficult task. There are many different kinds of optical aberrations and distortions in these systems. The influence of optical aberrations increases towards the edges (at a high angular distance from the optical axis of the system). These aberrations distort the PSF of an optical or imaging system, and rapidly reduce the accuracy of the measurements. The optical aberrations are dependent on the spatial data. They affect the transfer characteristics of the optical systems, and make them spatially variant 7 .

Astrometry measurements are often limited by variations of PSF shape and size over the image. These variations in PSF structure occur especially in UWFC systems, due to the number of aberrations, which increase towards the margins 7 .

Conventional methods in astrometry and photometry do not take into account the variance of PSF, and are therefore not suitable for wide-field image processing and scientific data measurements. When the PSF variations are known, the efficiency of these methods can be improved.

\section{IMAGE PROCESSING}

A wide-field astronomical image exposed by an MI G12000 camera with an ultra wide-angle lens in Namibia (southern Africa) was chosen for our investigation

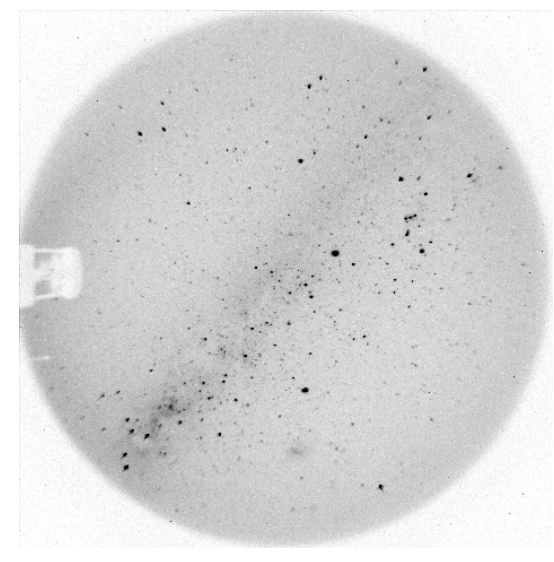

(a)

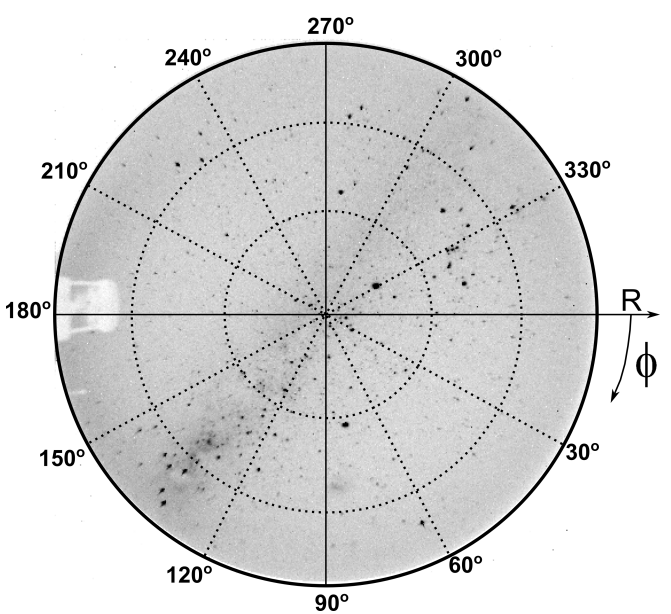

(b)

Figure 1. (a) Testing wide-field image. (b) Testing image split into regions. 

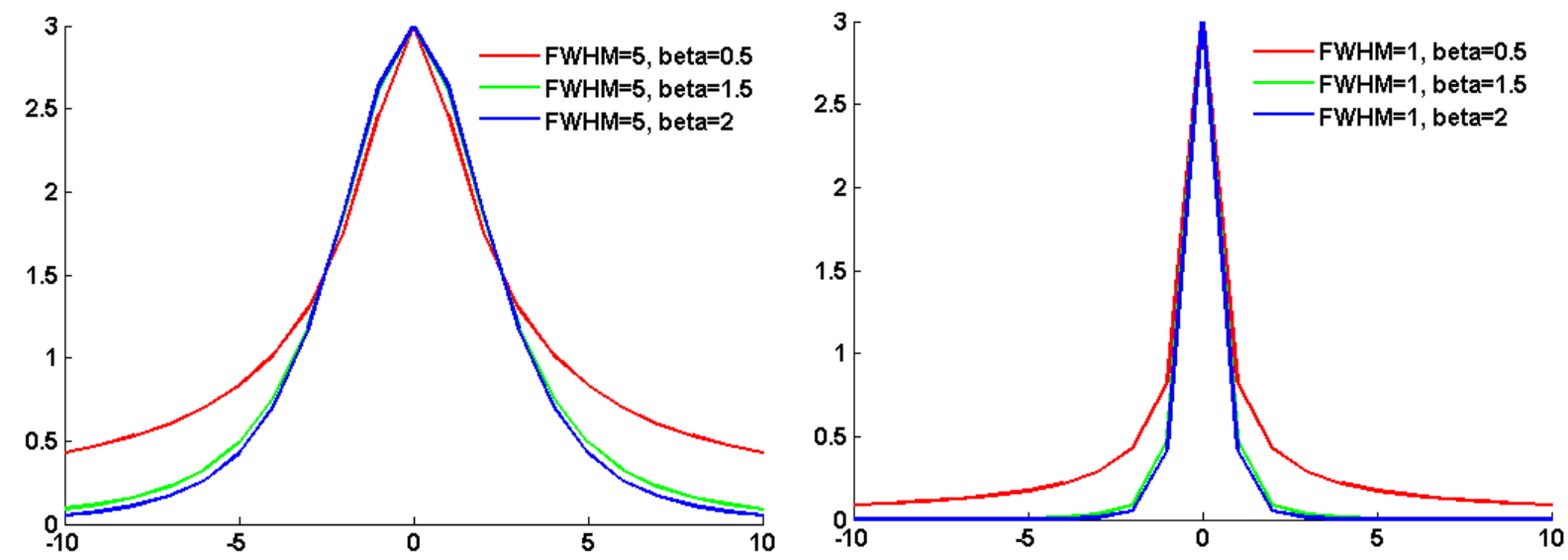

Figure 2. Moffat function shape depending on FWHM and atmospheric scattering coefficient $\beta$.

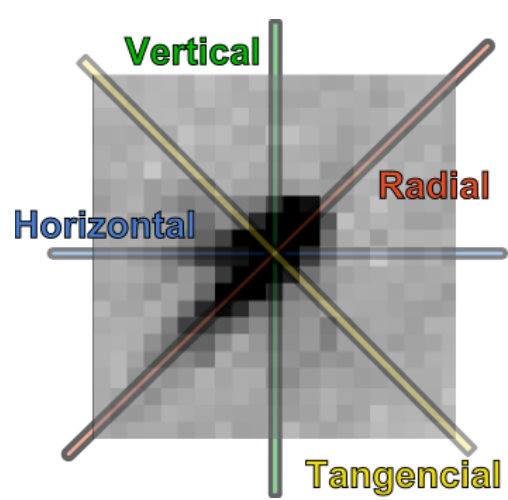

Figure 3. Moffat function fitting directions.

(Fig. 17). The processing of an astronomical image using MATLAB [6] is summarized below.

(1.) First, all stellar objects were detected using a detecting method [1, 2] based on the wavelet transform, realized by the à trous algorithm [8]. When the wavelet transform is implemented by the Mallat algorithm [5], there is for each additional degree of decomposition an image with dimensions twice smaller than on the previous level. This property is not good for stellar object detection, because it is appropriate to have the same number of pixels at each scale in order to comply with the same coordinates. Moreover, the objects in ultra widefield images are very small (a few pixels per object dimension), and they could be lost during dyadic decomposition. The wavelet transform is therefore realized by the à trous algorithm for astronomical purposes.

(2.) Then the stellar object coordinates were converted from Cartesian space $(x, y)$ to polar space $(R, \phi)$ in order to represent detected star distances from the center of the imaging system.

(3.) After that, the overall polar space was split into several areas in such a manner that each quadrant contains 9 regions, as shown in Fig. 11p. For simplification it was considered that the imaging system inside each region is space invariant (in other words,

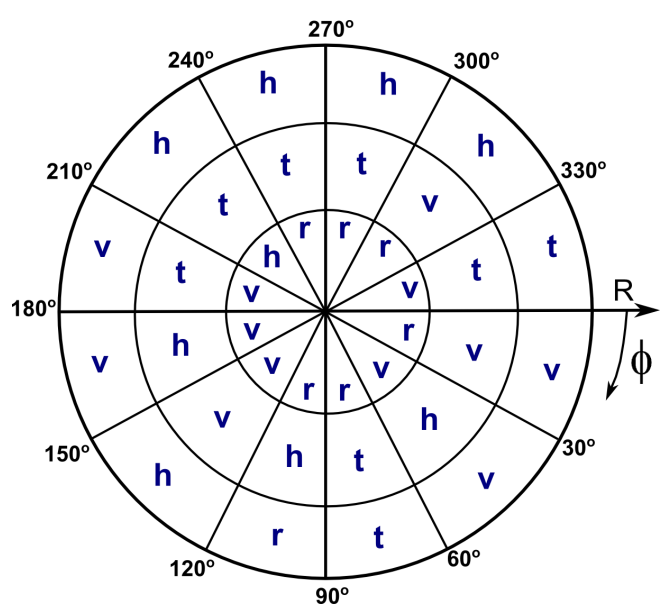

FiguRE 4. Fitting directions with the smallest difference between the chosen stellar shape and the Moffat function.

the imaging system has space invariant PSF in each region).

(4.) One brighter stellar object was selected in each area to represent the imaging system PSF on each position inside the image.

(5.) Moffat functions (1) with various FWHM (Full Width at Half Maximum) and atmospheric scattering coefficient $\beta$ parameters were fitted on selected stars within each region in horizontal, vertical, radial and tangential direction (Fig. 3) in order to investigate their shape. Figure 2 shows examples of Moffat function shapes.

$$
\begin{aligned}
I_{\text {moffat }} & =I_{\mathrm{m}}\left(1+\left(\frac{t}{\alpha}\right)^{2}\right)^{-\beta}, \\
\alpha & =\frac{\text { FWHM }}{2 \sqrt{2^{\frac{1}{\beta}}}-1},
\end{aligned}
$$

where $I_{\mathrm{m}}$ is the maximum of a Moffat function, $\beta$ is the atmospheric scattering coefficient, and $t$ represents the distance from the center of the object. 

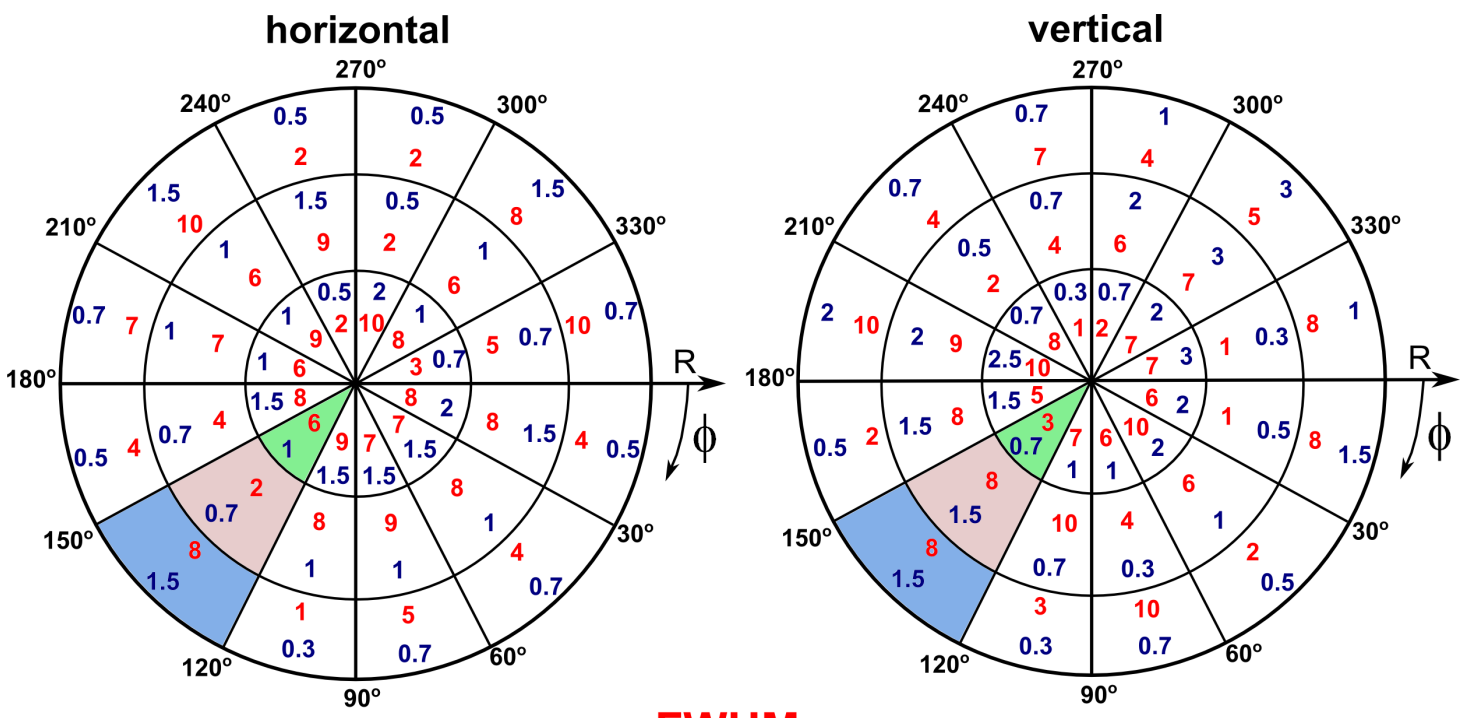

FWHM
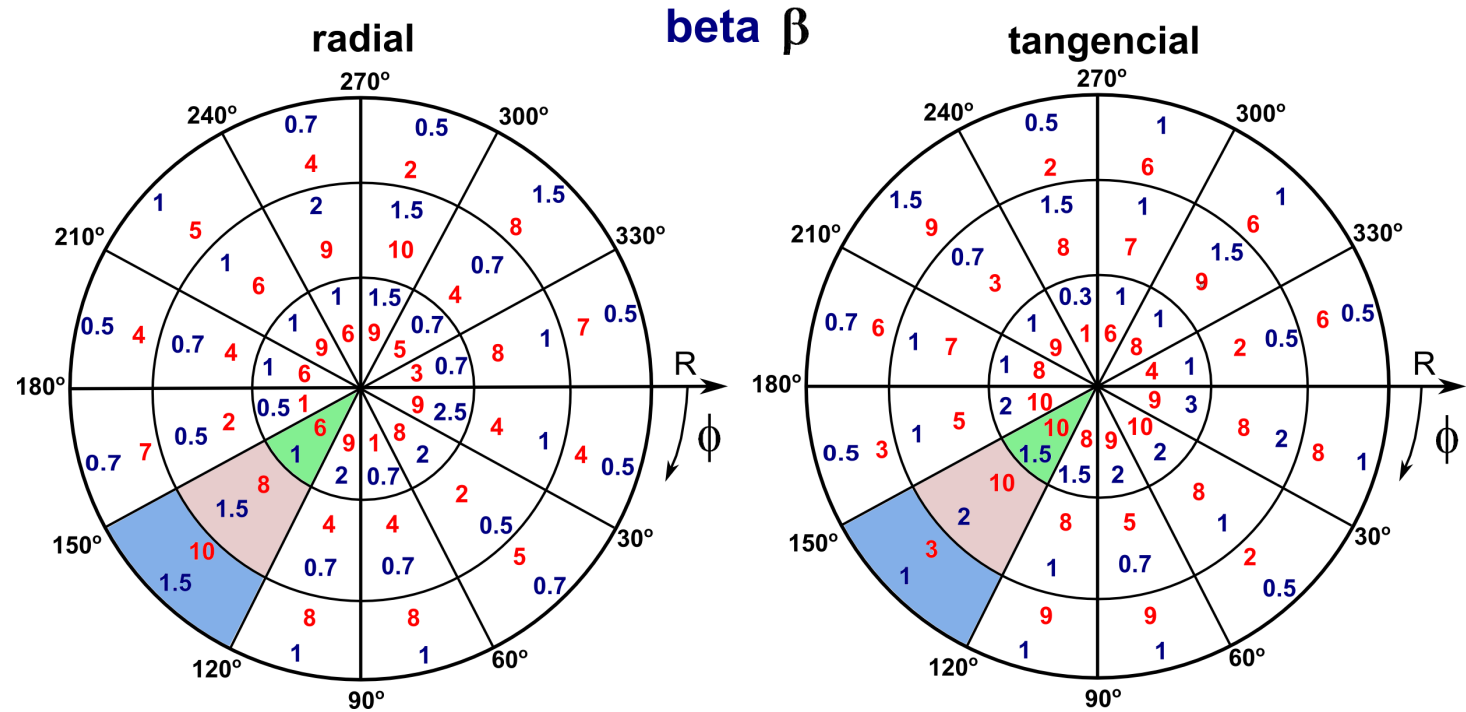

Figure 5. Moffat function fitting: FWHM and atmospheric scattering coefficient $\beta$ depending on stellar position and fitting direction.

\section{Results}

Figure 5 shows the fitting results: the dependence of the Moffat function parameters as FWHM and atmospheric scattering coefficient $\beta$ on the position inside the testing wide-field astronomical image and on the fitting direction. In the case of a perfectly round stellar object, the Moffat function parameters should be the same in all directions. As is shown in Fig. 5, this has been achieved only in a few cases. Correspondence of FWHM and $\beta$ in not more than two directions is a more common effect. Moreover, the FWHM and $\beta$ values of the Moffat function change in dependence on distance from the center of the astronomical image. This is due to the imaging system aberrations, as was mentioned above.

In addition, Fig. 4 shows the fitting directions that have the lowest difference between the chosen stellar shape and the Moffat function in each region. Vertical direction is marked as $\boldsymbol{v}$, horizontal direction as $\boldsymbol{h}$, radial direction as $\boldsymbol{r}$, and tangential direction as $\boldsymbol{t}$.

\section{Conclusions}

We have studied the spatial properties of the selected imaging system. We have confirmed that wide-field imaging systems have variable PSF. By a fitting process, we worked out the shape of the PSF in each area of the testing image. Figure 5 shows the fitting results: Moffat function parameters depending on distance from the center and on the spatial angle. However, note that each imaging system is different, so it is necessary to measure each system separately.

Moreover, stellar objects located at greater angular distances from the optical axis of the wide-field imaging system are not symmetrical in all surveyed directions (see Fig. 6). It is therefore more convenient to use an asymmetrical function for the fitting procedure, rather than a symmetrical Moffat function. We tried to fit stars in all directions using an asymmetrical function (3) which represents a Gaussian function in the logarithmic scale. However, it was found that the fitting results using this function 

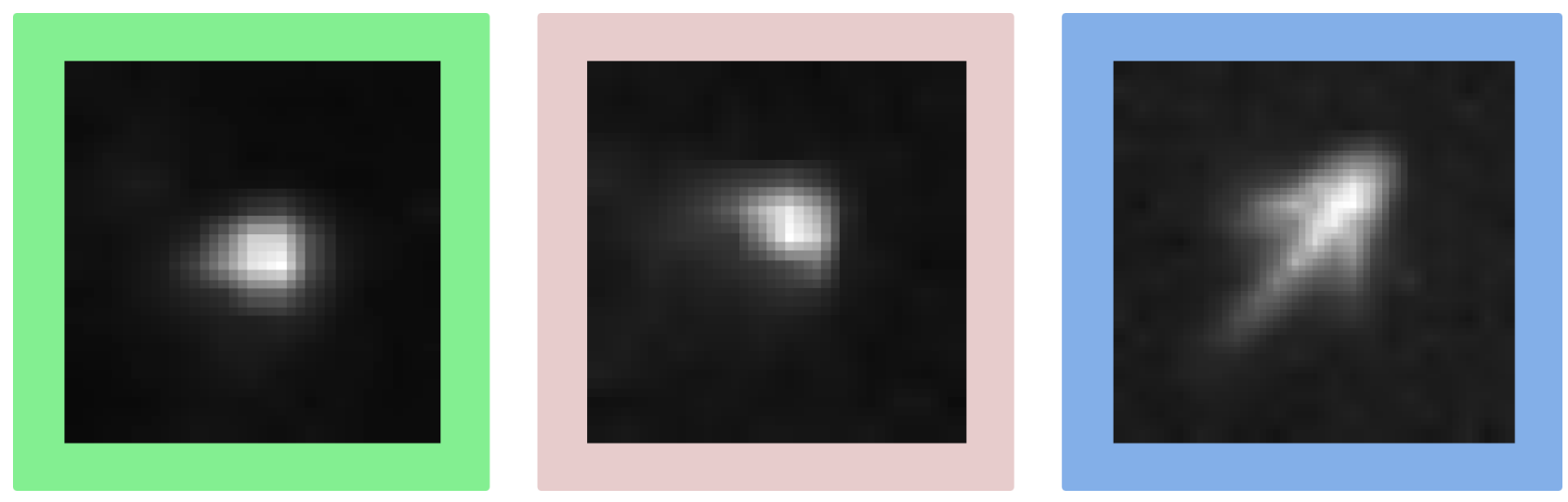

Figure 6. Examples of the shapes of stellar objects inside the colored regions in Fig. 5 .

(also in radial direction, where the PSF seems to be asymmetrical, see Fig. 6) are worse than when using symmetrical Moffat function. This means that we have to find some other asymmetrical function for the fitting procedure.

$$
I_{\text {gauss-log }}(x)=I_{\mathrm{m}}\left(e^{\frac{-\log ^{2} x}{2 \sigma^{2}}}\right)
$$

Our goal is to improve the effectiveness of astrometric and photometric algorithms with more knowledge of the spatially variant PSF shape, e.g. algorithms for stellar object detection and profile photometry. Our plans include enhancing a stellar object detecting algorithm using the wavelet transform [1, 2] with a spatially variant wavelet function. We would also like to use the properties of Zernike polynomials [4] to reconstruct the impaired light wavefront. However, it is necessary to reduce the noise before image processing and scientific data measurement. In this area, papers [9 11] can provide inspiration for our future work.

\section{ACKNOWLEDGEMENTS}

This paper has been supported within the framework of project SGS10/285/OHK3/3T/13 "Analysis of spatially and time variable scientifical imaging systems" of the CTU Student grant competition.

Authors would like to thank the Institute of Physics CSAS for providing the astronomical images from FULLSKY camera in Namibia.

\section{REFERENCES}

[1] E. Anisimova. Methods for Analysing and Processing of Astronomical Image Data. Thesis. Czech Technical University in Prague, Faculty of Electrical Engineering, Department of Radio Engineering, Prague, 2011, 77pp.
[2] E. Anisimova. P. Páta, M. Blaźek. Stellar Object Detection Using the Wavelet Transform. Acta Polytechnica 2011, vol. 51, no. 6, pp 9-12.

[3] D. L. Donoho, I. M. Johnstone. Ideal Spatial Adaptation by Wavelet Shrinkage. Biometrika 1994, vol. 81, pp. 425-455.

[4] J. A. Díaz, J. Fernández-Dorado, C. Pizarro, J. Arasa. Zernike Coefficients for Concentric, Circular, Scaled Pupils: An Equivalent Expression. Journal of Modern Optics 2009, vol. 56, no. 1, pp. 149-155.

[5] S. G. Mallat. A Theory for Multiresolution Signal Decomposition: The Wavelet Representation. IEEE Transactions on Pattern Analysis and Machine Intelligence 1989, vol. 11, no. 7 .

[6] MATLAB. The Language of Technical Computing http://www . mathworks.com/products/matlab/ [2012-08-27].

[7] M. Řeřábek, P. Páta, K. Fliegel, et al. Space variant point spread function modeling for astronomical image data processing. Astronomical Adaptative Optics Systems and Applications III, Proceedings of the Society of Photo-optical Instrumentation Engineers (SPIE), vol. 6691, pp. T6910-T6910, 2007.

[8] J.-L. Starck, F. Murtagh. Astronomical Image and Data Analysis Springer-Verlag Berlin Heidelberg, 2002. 289 pp..

[9] J. Švihlík. Modeling of Scientific Images Using GMM. Radioengineering 2009, vol. 18 , no. 4, pp. 579-586.

[10] J. Švihlík, K. Fliegel, P. Koten. Noise Analysis of MAIA System and Possible Noise Suppression. Radioengineering 2011, vol. 20, no. 1, pp. 110-117.

[11]J. Švihlík, K. Fliegel, J. Kulal, et al. Estimation of non-Gaussian noise parameters in the wavelet domain using the moment-generating function. Journal of Electronic Imaging 2012, vol. 21, article no. 023025. 